\title{
Temporal Bayesian Networks for Scenario Recognition
}

\author{
Ahmed Ziani and Cina Motamed \\ Laboratoire LASL EA 2600 \\ Université du Littoral Côte d'Opale \\ Bat 2, 50 Rue F.Buisson \\ 62228 Calais France \\ ziani@lasl.univ-littoral.fr, motamedalasl.univ-littoral.fr
}

\begin{abstract}
This work presents an automatic scenario recognition system for video sequence interpretation. The recognition algorithm is based on a Bayesian Networks approach. The model of scenario contains two main layers. The first one enables to highlight atemporal events from the observed visual features. The second layer is focused on the temporal reasoning stage. The temporal layer integrates an event based approach in the framework of the Bayesian Networks. The temporal Bayesian network tracks lifespan of relevant events highlighted from the first layer. Then it estimates qualitative and quantitative relations between temporal events helpful for the recognition task. The global recognition algorithm is illustrated over real indoor images sequences for an abandoned baggage scenario.
\end{abstract}

Keywords: Visual-surveillance, scenario recognition, image sequence analysis, Bayesian Network.

\section{Introduction}

The ability to automatically resume and index video content is an important challenge of the vision and data base communities. The motion interpretation of video is an important area of investigation. Indeed in MPEG-7 standard, a set of general motion descriptors has been defined: camera motion, object motion trajectory, parametric object motion and motion activity.

The purpose of this work is the recognition of specific scenarios of human activity in visual surveillance applications [1]. For such dynamic scenes, scenarios are based on a combination of spatial, temporal and interacting events. This automatic recognition is firstly helpful for video-surveillance operator for an on-line alarm generation by highlighting abnormal situation. The second utility concerns the off-line retrieval of specific behaviours from a stored image sequence. This capability becomes naturally more powerful when the monitoring implies several cameras working simultaneously.

From a video indexing point of view, the specificity of this application with respect to general video sequences is that camera parameters and the majority of the observed background remain fixed. These favourable conditions permit to focus the attention on the object motion. 
A video surveillance system generally contains three main hierarchical stages. The motion detection, the tracking stage, and finally, the high level motion interpretation. The main objectives are to observe the scene, index activities and recognise the modelled scenarios.

Scenarios concern specific spatio-temporal trajectory, interaction between static or non static objects, or also a combination of both of them.

\section{Motion Detection and Tracking}

The detection of moving objects is then made by comparing the three RGB components:

$$
\text { if } \begin{gathered}
\left(\max _{c=R, G, B}\left|I_{c}^{k}(P)-R_{c}^{k-1}(P)\right|>\omega\right) \\
\rightarrow D^{k}(P)=1 \\
\text { else } \rightarrow D^{k}(P)=0
\end{gathered}
$$

$\mathrm{D}^{\mathrm{k}}$ represents the detection decision (1: moving object, 0 : background) and $\mathrm{R}^{\mathrm{k}}$. the reference images. The raw motion detection result generally presents many artefacts. Two kinds of cleaning procedures are applied. Firstly, we use standard morphological operations of erosion and dilatation for reducing noise in the foreground. Then, too small uninteresting image regions are removed. In our algorithm this size threshold is defined globally in an empirical manner for the entire image. The Figure 1 illustrates a motion detection result for a real sequence of human activity.

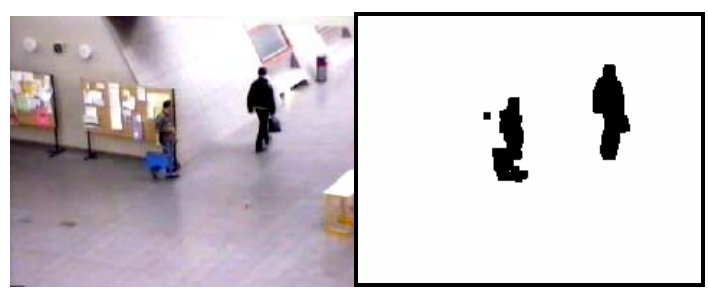

Fig. 1. Detection process: $\mathrm{I}^{\mathrm{k}}$ and $\mathrm{D}^{\mathrm{k}}$

The tracking process matches the detected regions from a temporal sequence to another and takes into account the splitting and merging phenomenon during object motion. One important component of our tracking algorithm is the integration of a belief revision mechanism associated with each detected object, reflecting an instantaneous quality of its tracking [2]. We consider that the belief can increase when:

- The tracked object follows a continuous path;

- The object size is stable;

- No ambiguity appears (other target meeting: target splitting, loss of target...); Otherwise the belief decreases. 


\section{Scenario Recognition}

A Scenario is composed of a set of elementary events linked with constraints. These links represent the ordering of the events or temporal constraints. Several kinds of events can be used, and depend directly on the observed scene and on the objective of the surveillance task.

The difficulty of human activity scenarios is their variabilities. This variability can be both spatial, and behavioural. So the scenario recognition process must be able to handle such uncertainties.

The logical structure linking events is generally based on graph. Many approaches are used: Petri-net [3] Bayesian network [4], [5], Network of time constraints also brings an efficient way to represent temporal structures of scenarios [6]. HHM are a popular state based probabilistic approach for representing dynamic systems, they have been initially used in speech recognition and have been successfully applied over gesture recognition [7]. An interesting feature of HMM is its time scale invariance enabling scenarios in various speeds. The Couple HHM has also been developed in order to efficiently represent interaction between objects [4]. But the major disadvantage of the HMM approaches is their complexities and the need of a long learning step in order to adjust model parameter. In this work, the model of scenarios is also obtained with the Bayesian networks formalism.

\subsection{Bayesian Networks, Definitions}

A Bayesian network is a directed acyclic graph whose:

- Nodes represent variables;

- Arcs represent dependence relations between the variables and local probability distributions for each variable given values of its parents.

Nodes have the possibility to represent any kind of random or determinist variables. They can be attached with a measured parameter or a latent variable.

Bayesian Networks generally operate on discrete values of nodes but can also deals with continuous representation.

If there is an arc from node $\mathrm{A}$ to another node $\mathrm{B}$, then variable $\mathrm{B}$ depends directly on variable $A$, and $A$ is called a parent of $B$. If for each variable $X_{i}, i=1$ to $n$, the set of parent variables is denoted by parents $X_{i}$ ) then the joint distribution of the variables is product of the local distributions

$$
P\left(X_{1}, \ldots, X_{n}\right)=\prod_{i=1}^{n} P\left(X_{i} \mid \operatorname{parents}\left(X_{i}\right)\right)
$$

One important advantage of the Bayesian network is its ability to encode qualitative and quantitative contextual knowledge and their dependence and it brings an efficient inference structure for real time application [8]. The topology of the network represents a qualitative causal relation between variable and the joint probability represents the quantitative part of the network. 
The joint probability is represented by the expression

$$
P\left(X_{j}, \mathrm{H}_{i}\right)=P\left(X_{j} \mid \mathrm{H}_{i}\right) P\left(\mathrm{H}_{i}\right)
$$

Then the objective is to estimate hypotheses $H_{i}(i=1, . ., N)$ based on evidence $X_{j}$

$$
P\left(\mathrm{H}_{i} \mid X_{j}\right)=\frac{P\left(X_{j}, \mathrm{H}_{i}\right)}{P\left(X_{j}\right)}
$$

One of the major difficulties of using Bayesian Networks approach concerns the design of the network structure. In fact every dependency must be taken into account in order to make a good representation of the problem.

\subsection{Bayesian Networks and Visual Scenario Recognition}

Bayesian networks have been widely used in computer vision community for event or scenario recognition. The system described by [5] delivers textual descriptions for dynamic activities occurring in a dynamic, containing vehicles and pedestrians. [9], used Bayesian Networks to recognize several activities in an American football game. [10], suggested that for events with logical and temporal relationships between them, Allen's relations can be used to describe temporal relations between sub-events. A particular form of dynamic Bayesian networks, Recurrent Bayesian Networks (RBNs), have been used for the recognition of human behaviours [11]. Such networks have the advantage to have some time scale independence.

In order to model efficiently general scenarios, the system has to model temporal constraint between elementary events. The time aspect in Bayesian Network has led to several approaches.

The first common category is known as "time slice" approach. The main technique is the Dynamic Bayes networks [12], [13]. It assumes a Markov property by considering that a single snapshot in the past is sufficient for predicting the future. The structure of a static $\mathrm{BN}$ is generated for a specific instant and repeated with the same structure over the time. Temporal arc are added between nodes belonging to different time instants.

The second category, less explored, represents the "event based" approach which permits to integrate explicitly temporal nodes (Temporal Nodes Bayesian Networks (TNBN) [14] and Net of Irreversible Events in Discrete Time (NIEDT) [15]. This second category of approaches has several advantages. Firstly, it is particularly adapted when the system has to manipulate the notion of time at several temporal granularities. Secondly, the event based approach can easily establish quantitative temporal relation between events as Allen interval [16]. In time slice approach there is no way to represent naturally concept as an event $\mathrm{e}_{1}$ appears before another event $\mathrm{e}_{2}$. The HMM approach can be seen as a particular case of DBN and consequently shares the same limitations. 


\subsection{Proposed Model}

In this work we propose a scenario recognition algorithm based on an "event based" Bayesian Network approach. The global structure of our proposed network uses the concept of Hierarchical Bayes networks that have been proposed as a technique of incorporating a Bayesian Network inside a node of a higher level Network. Such encapsulation facilitates the hierarchical decomposition of the problem into subproblem.

The model of the scenario is composed by two layers: the atemporal event layer and the temporal reasoning layer. Is such organisation the first layer contains low level networks representing each atemporal event.

Each atemporal event is represented a hierarchical Bayesian network. The lower level is linked with observed data $\mathrm{X}_{\mathrm{j}}$ and the upper level represents an hypothesis $\mathrm{H}_{\mathrm{i}}$ $(\mathrm{i}=1, \ldots, \mathrm{N})$ representing latent nodes. In our problem, observed nodes are measured visual features $\mathrm{X}$ and the hypotheses $\mathrm{H}$ represent decision of the elementary events recognition. Visual features used at this layer are: positions, speed, direction and size of tracked objects.

The temporal reasoning layer is based on a set of Bayesian networks which contain nodes associated with temporal information. Such nodes generally use the result obtained by estimating the lifespan of hypotheses delivered by the atemporal event layer. First of all, the objective of the temporal layer is to evaluate qualitative or quantitative temporal constraints for each event and also to estimate relations between related events. Constraints represent the duration or the time of occurrence (relative or absolute) of an event. Relations between events represent their mutual temporal dependency (before, after, during etc..). The low level temporal nodes have to detect the "Start" and "End" time of their events of interest. We have chosen to propagate explicitly the uncertainty of these last estimations over the scenario recognition task. For this, each date is approximated by a normal distribution with respect to its mean and variance values. Then, Allen relationships between events are implemented by using specific nodes in order to evaluate temporal relations between events. This network contains nodes which compute some hypothesis linked with the start and end times of events (Table 1). The $\mathrm{Ds}_{1} \mathrm{~s}_{2}$ is the distance of the start time of event 1 and the start time of the event 2 . The $\mathrm{Ds}_{1} \mathrm{e}_{2}$ is the distance of the start time of event 1 and the end time of the event 2 . The use of Bayesian network at this layer is efficient for tacking into account uncertainty of estimated dates (start and end time of event). The general structure of the Bayesian network which has to verify the temporal relations is defined in the (Fig.2).

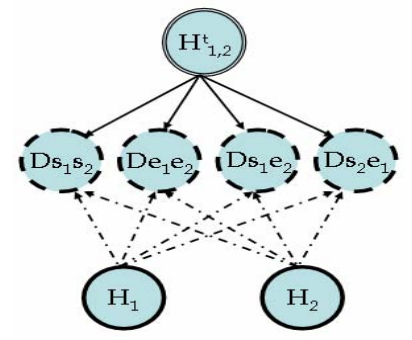

Fig. 2. Model of temporal relations
Table 1. Example of the relation 'Equal' of allen

\begin{tabular}{|c|}
\hline $\mathrm{H}_{1}$ equal $\mathrm{H}_{2}$ \\
\hline$D_{s_{1} s_{2}}$ true if $\left(s_{2}-s_{1}=0\right)$ \\
$D_{e_{1} e_{2}}$ true if $\left(e_{2}-e_{1}=0\right)$ \\
$D_{s_{1} e_{2}}$ true if $\left(e_{2}-s_{1}>0\right)$ \\
$D_{s_{2} e_{1}}$ true if $\left(e_{1}-s_{2}>0\right)$ \\
\hline
\end{tabular}


The integration of the temporal constraints associated with an event is also performed with this approach, by linking observed events and with constraints which are represented by specific nodes as time point or duration (relative or absolute timebase). Generally the constraints defined from the contextual information of the scenario are defined by a relative time base with respect to other previous events. The figure 3 illustrates the general structure of the suggested scenario model.

Table 2. Various situations of the relationships of allen

\begin{tabular}{|l|c|c|c|c|}
\hline \multicolumn{1}{|c|}{ Relations } & $\mathrm{Ds}_{1} \mathrm{~s}_{2}$ & $\mathrm{De}_{1} \mathrm{e}_{2}$ & $\mathrm{Ds}_{1} \mathrm{e}_{2}$ & $\mathrm{Ds}_{2} \mathrm{e}_{1}$ \\
\hline $\mathrm{H}_{1}$ starts $\mathrm{H}_{2}$ & $\mathrm{Ds}_{1} \mathrm{~s}_{2}=0$ & $\mathrm{De}_{1} \mathrm{e}_{2}<0$ & $\mathrm{Ds}_{1} \mathrm{e}_{2}>0$ & $\mathrm{Ds}_{2} \mathrm{e}_{1}>0$ \\
\hline $\mathrm{H}_{1}$ finishes $\mathrm{H}_{2}$ & $\mathrm{Ds}_{1} \mathrm{~s}_{2}>0$ & $\mathrm{De}_{1} \mathrm{e}_{2}=0$ & $\mathrm{Ds}_{1} \mathrm{e}_{2}>0$ & $\mathrm{Ds}_{2} \mathrm{e}_{1}>0$ \\
\hline $\mathrm{H}_{1}$ during $\mathrm{H}_{2}$ & $\mathrm{Ds}_{1} \mathrm{~s}_{2}>0$ & $\mathrm{De}_{1} \mathrm{e}_{2}<0$ & $\mathrm{Ds}_{1} \mathrm{e}_{2}>0$ & $\mathrm{Ds}_{2} \mathrm{e}_{1}>0$ \\
\hline $\mathrm{H}_{1}$ equal $\mathrm{H}_{2}$ & $\mathrm{Ds}_{1} \mathrm{~s}_{2}=0$ & $\mathrm{De}_{1} \mathrm{e}_{2}=0$ & $\mathrm{Ds}_{1} \mathrm{e}_{2}>0$ & $\mathrm{Ds}_{2} \mathrm{e}_{1}>0$ \\
\hline $\begin{array}{l}\mathrm{H}_{1} \text { meets } \mathrm{H}_{2} \\
\mathrm{H}_{1} \text { before } \mathrm{H}_{2}\end{array}$ & $\mathrm{Ds}_{1} \mathrm{~s}_{2}>0$ & $\mathrm{De}_{1} \mathrm{e}_{2}>0$ & $\mathrm{Ds}_{1} \mathrm{e}_{2}>0$ & $\mathrm{Ds}_{2} \mathrm{e}_{1}=0$ \\
\hline $\mathrm{H}_{1}$ overlaps $\mathrm{H}_{2}$ & $\mathrm{Ds}_{1} \mathrm{~s}_{2}>0$ & $\mathrm{De}_{1} \mathrm{e}_{2}>0$ & $\mathrm{Ds}_{1} \mathrm{e}_{2}>0$ & $\mathrm{Ds}_{2} \mathrm{e}_{1}>0$ \\
\hline
\end{tabular}

For illustration, in (Fig. 4), we present the model of a scenario of an abandoned baggage by using the proposed approach. $\mathrm{H}_{12}^{\mathrm{t}}$ represents temporal node verifying relation between events $\mathrm{H}_{1}$ and $\mathrm{H}_{2}$. The node $\mathrm{H}_{3}^{\mathrm{t}}$ in the temporal layer permits to verify a temporal duration of the $\mathrm{H}_{3}$ with a constraint of a defined duration $(\Delta \mathrm{t})$.

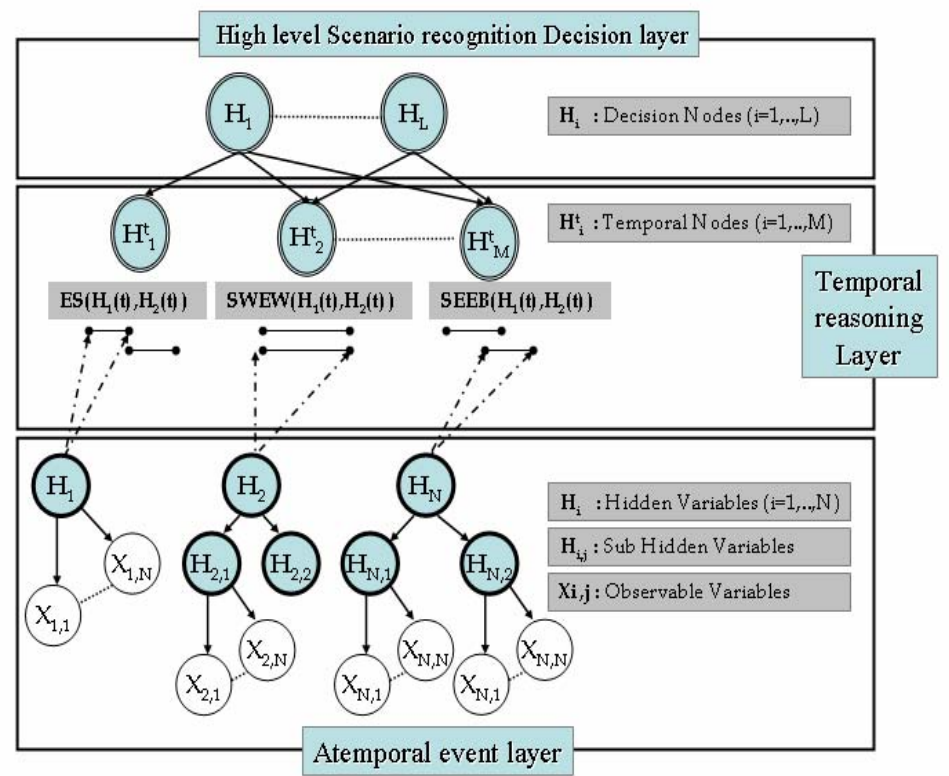

Fig. 3. General model of a scenario 


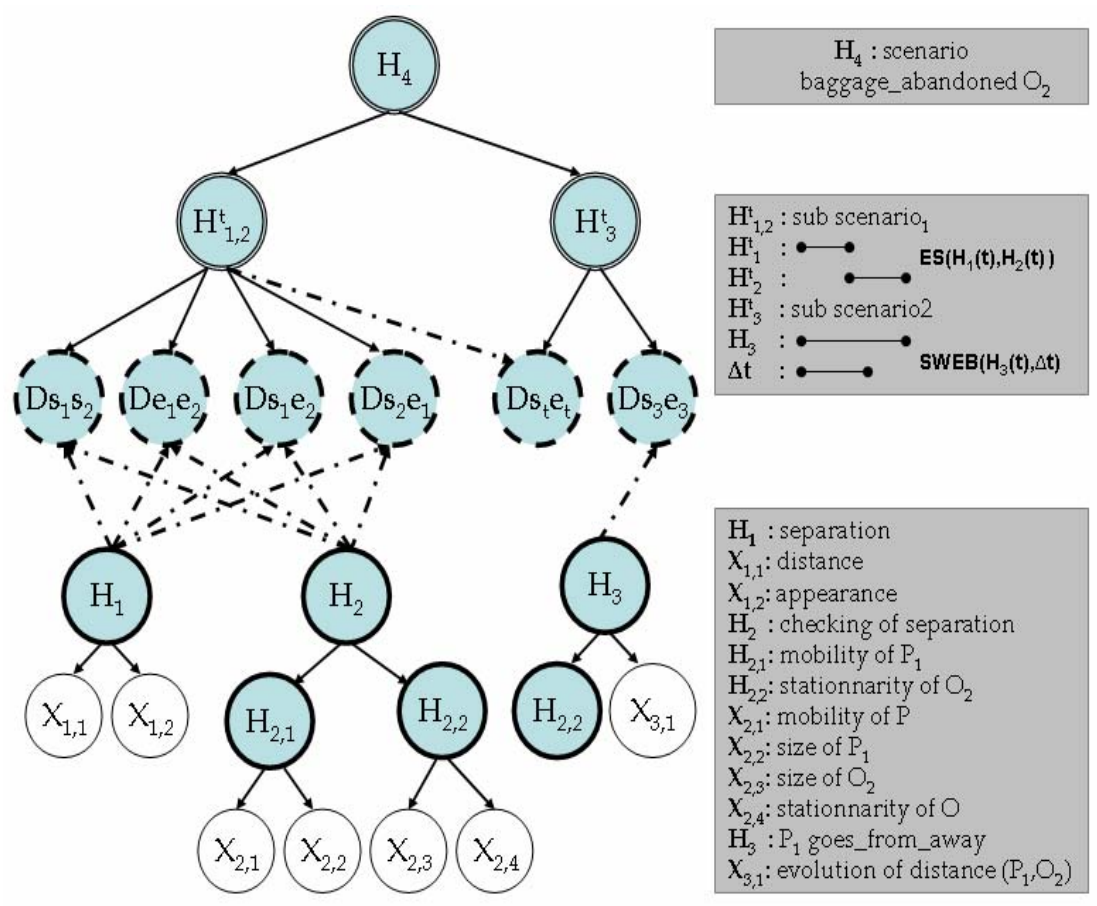

Fig. 4. Model of the scenario for an abandoned baggage

\subsection{Parameters Learning}

Generally, it is possible to learn the network parameters, from the experimental data, in particular the conditional probabilities tables.

$$
P\left(X_{i}=x_{k} \mid \operatorname{paren}\left(X_{i}\right)=c_{j}\right)=\theta_{i, j, k}=\frac{n_{i, j, k}}{\sum_{K} n_{i, j, k}}
$$

Where $n_{i, j, k}$ is the number of the events in the database for which variable $X_{i}$ is in the state $\mathrm{x}_{\mathrm{k}}$ and his parents are in the configuration $\mathrm{c}_{\mathrm{j}}$.

In the context of visual surveillance, it is not always realistic to perform the standard learning procedure. Because, some times, it's not possible to have sufficient occurrences for each scenario. Another difficulty is that learning process has to deal with uncertain inputs. Parameters for a fixed network from incomplete data, in the presence of missing values or hidden variables can be estimated by the EM Expectation-Maximisation algorithm.

We illustrate the learning process by using the EM over an example from the abandoned baggage scenario (Fig. 5). The incomplete data are presented in Table 3.

The EM algorithm is initialized with $\mathrm{P}^{(0)}\left(\mathrm{H}_{2,1}=1\right)=0.5$ and $\mathrm{P}^{(0)}\left(\mathrm{H}_{2,1}=0\right)=0.5$. Convergence is obtained after $13^{\text {th }}$ iterations: $\mathrm{P}^{(13)}\left(\mathrm{X}_{2,1}=1 \mid \mathrm{H}_{2,1}=1\right)=0.857$ and $\mathrm{P}^{(13)}\left(\mathrm{X}_{2,2}=1 \mid \mathrm{H}_{2,1}=0\right)=0.985$. The figure 6 shows the evolution of these probabilities. 


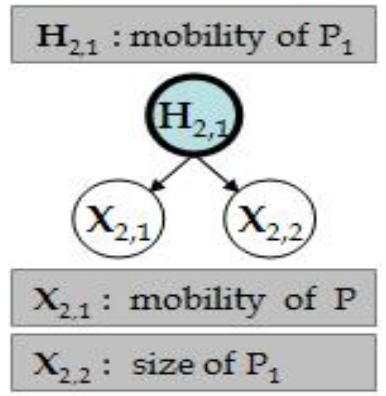

Table 3. Table of occurrence

\begin{tabular}{|c|c|c|c|}
\hline $\mathbf{X}_{2,1}$ & $\mathbf{X}_{2,2}$ & $\mathrm{H}_{2,1}$ & $\mathrm{Nb}$ \\
\hline 1 & 1 & 1 & 18 \\
\hline 1 & 1 & 0 & 1 \\
\hline 0 & 1 & 0 & 3 \\
\hline 1 & 0 & 0 & 2 \\
\hline$?$ & 1 & 1 & 1 \\
\hline 1 & $?$ & 1 & 2 \\
\hline 0 & 0 & 0 & 14 \\
\hline 0 & $?$ & 0 & 3 \\
\hline
\end{tabular}

Fig. 5. Learning of a naive bayesian network, an example

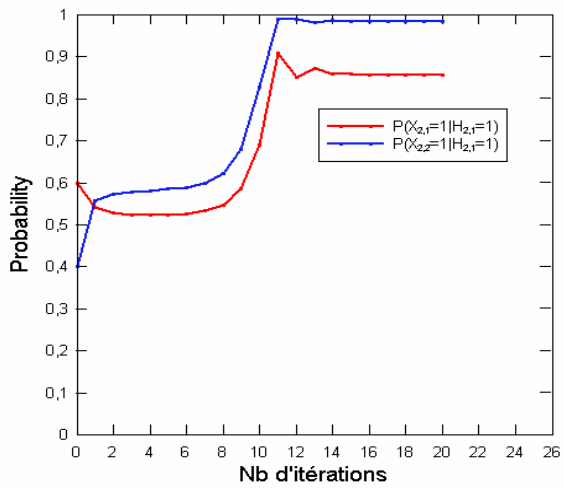

Fig. 6. Evolution of the estimated probabilities

\section{Results}

Two experimentations based on real indoor sequences are presented in this section. Both sequences represent a scenario of abandoned baggage. The first sequence is obtained from the scene that we have used for learning of the scenario model (Fig. 7). The second sequence comes from the PETS'04 dataset (Fig. 8). The results illustrate the evolution of the main nodes of the scenario recognition model.

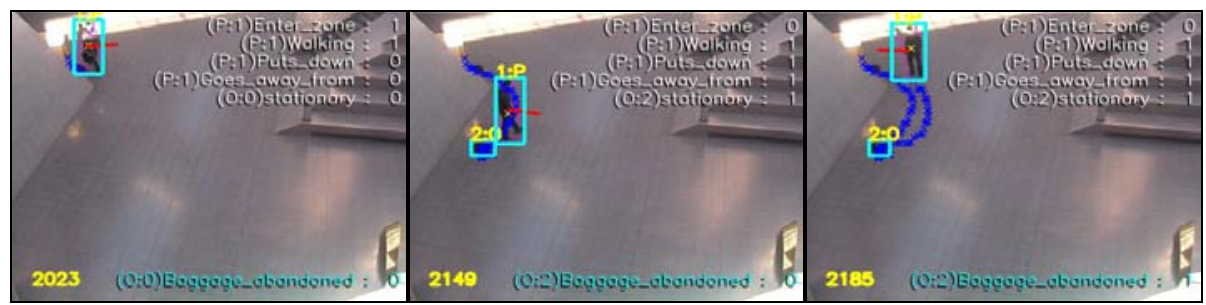

Fig. 7. First sequence 


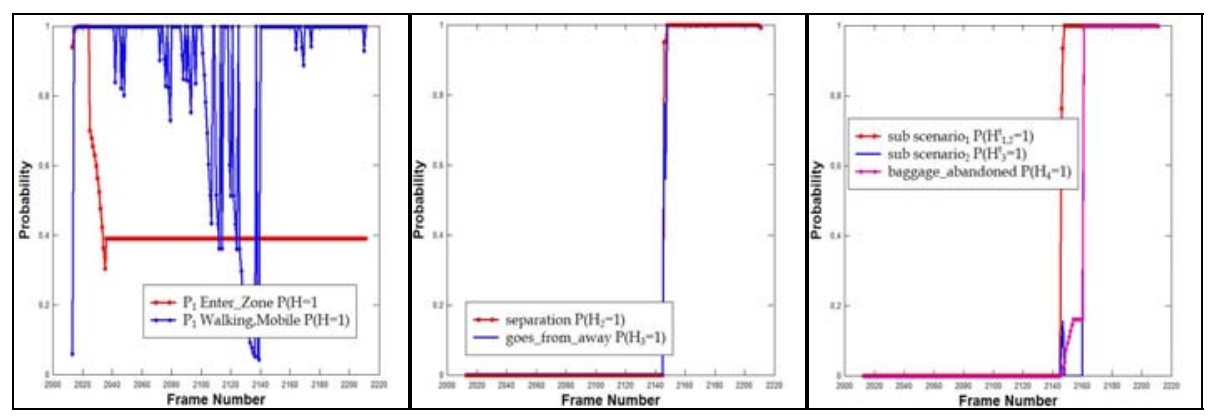

Fig. 7. (Continued)

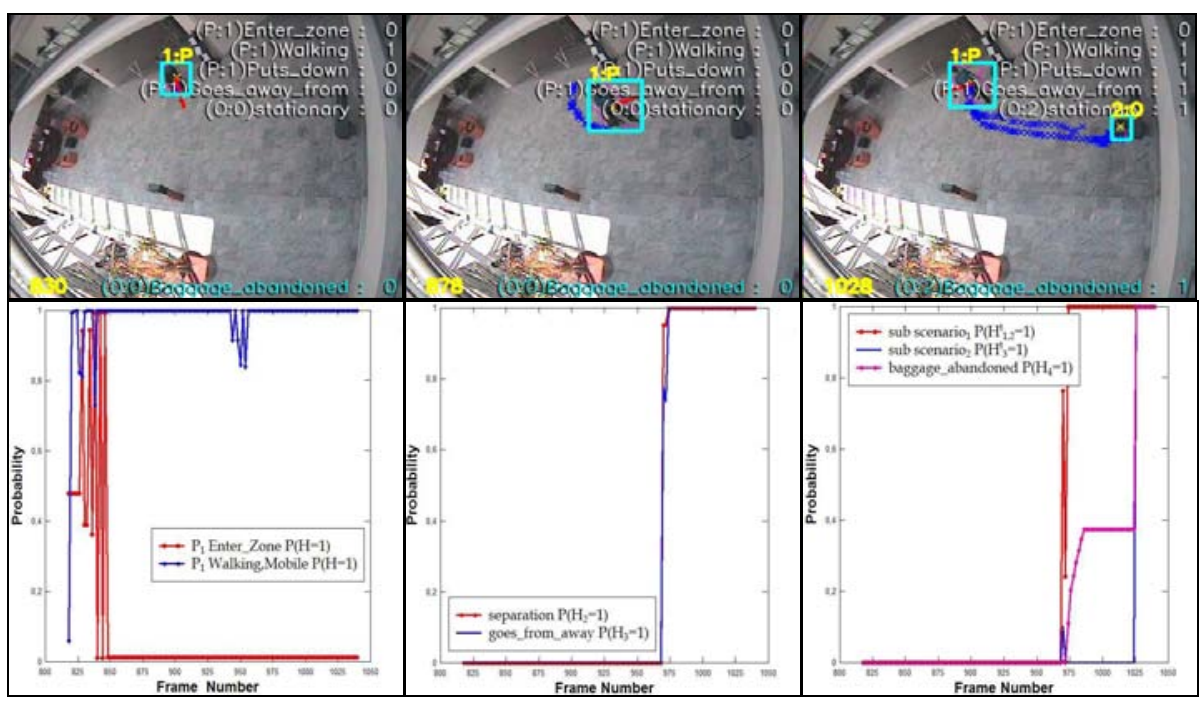

Fig. 8. Sequences from PETS'04 Workshop

\section{Conclusions}

The proposed scenario recognition algorithm is based on the Bayesian network. It permits to deal efficiently with uncertainty due to the low level visual extraction stage and also the variability of scenario models. The model contains two main layers. The first one permits to highlight events from the observed visual features. The second layer is focused on the temporal reasoning stage by using specific nodes based on the temporal validity of the first layer's events. The temporal reasoning permits firstly to estimate the relation between events and also to add quantitative and qualitative on the high level recognition decision. The use of node containing temporal information under the framework of the Bayesian Networks permits to have a flexible temporal reasoning capability over the recognition task. The separation of the scenario model by two complementary layers brings many advantages as modularity and clarity. The specificity of our temporal layer with respect to other event based approaches is that it 
can deal easily with all kind of constraints or relation (qualitative, quantitative, relative and absolute). In fact, the majority of existing event based approaches use fixed quantitative time interval. The global recognition algorithm is tested and validated on real indoor images sequences.

\section{References}

1. Buxton, H.: Learning and Understanding Dynamic Scene Activity: A Review. Image and Vision Computing 21, 125-136 (2003)

2. Motamed, C.: Motion detection and tracking using belief indicators for an automatic visual- surveillance system. Image and Vision Computing 24(11), 1192-1201 (2006)

3. Castel, C., Chaudron, L., Tessier, C.: What Is Going On? A High Level Interpretation of Sequences of Images. 4th European conference on computer vision, Workshop on conceptual descriptions from images,Cambridge UK (1996)

4. Oliver, N., Rosario, B., Pentland, A.: A Bayesian Computer Vision System for Modeling Human In-teractions. In: Christensen, H.I. (ed.) ICVS 1999. LNCS, vol. 1542, Springer, Heidelberg (1998)

5. Remagnino, P., Tan, T., Baker, K.: Agent orientated annotation in model based visual surveillance, Proceedings of the International Conference on Computer Vision, pp. 857$862(1998)$

6. Eude, V., Bouchon-Meunier, B., Collain, E.: Reconnaissance d'activités à l'aide de graphes temporels flous. In: Proc LFA'97 Logique Floue et Applications, Lyon France pp. 91-98 (1997)

7. Starner, T., Pentland, A.: Real-time American Sign Language Recognition from Video Using Hidden Markov Models. In: Proceedings of International Symposium on Computer Vision, pp. 265-270 (1995)

8. Pearl, J.: Probabilistic reasoning in Intelligent Systems. Morgan Kaufman, San Francisco (1988)

9. Intille, S.S., Bobick, A.F.: Visual Recognition of multi-agent Action using Binary Temporal Relations. In: IEEE Proceedings of Computer Vision and Pattern Recognition, Fort Collins, CO (1999)

10. Hongeng, S., Nevatia, R.: Multi-Agent Event Recognition, ICCV ‘01, pp. 84-93 (2001)

11. Moenne-Loccoz, N., Bremond, F., Thonnat, M.: Recurrent Bayesian Network for the Recognition of Human Behaviors from Video. In: Crowley, J.L., Piater, J.H., Vincze, M., Paletta, L. (eds.) ICVS 2003. LNCS, vol. 2626, pp. 68-77. Springer, Heidelberg (2003)

12. Dean, T., Kanazawa, K.: A model for reasoning about persistence and causation. Computational Intelligence 5(3), 142-150 (1989)

13. Nicholson, A., J.M., B.: The data association problem when monitoring robot vehicles using dynamic belief networks. In: ECAI 92: 10th European Conference on Artificial Intelligence (1992)

14. Arroyo-Figueroa, G., Sucar, L.E.: A Temporal Bayesian Network for Diagnosis and Prediction. Uncertainty in artificial intelligence. In: Proc15th Conf Uncertainty Artif Intell pp. 13-20 (1999)

15. Galan, S.F., Diez, F.J.: Modeling dynamic causal interactions with bayesian networks: temporal noisy gates. In: CaNew', the 2nd International Workshop on Causal Networks held in conjunction with ECAI 2000, Berlin, Germany, pp.1-5 (August 2000)

16. Allen, J.F.: An interval based representation of temporal knowledge. International Joint Conference on Artificial Intelligence 81, 221-226 (1981)

17. Koller, D., Pfeiffer, A.: Object-oriented Bayesian networks. In: Koller, D., Pfeiffer, A. (eds.) Uncertainty in Artificial Intelligence: Proceedings of the Thirteenth Conference (UAI-1997), pp. 302-313. Morgan Kaufmann Publishers, San Francisco (1997) 